\title{
Two-Dimensional Measurement of Electron Density Profile in the Edge Region of the Large Helical Device Plasma by a Sheet-Shaped Thermal Lithium Beam
}

\author{
Yoshiyuki TAKAHASHI, Tomohiro MORISAKI ${ }^{1)}$, Kazuo TOI ${ }^{1)}$ and LHD Experimental Group ${ }^{1)}$ \\ Department of Energy Engineering and Science, Nagoya University, Nagoya 464-8603, Japan \\ ${ }^{1)}$ National Institute for Fusion Science, Toki 509-5292, Japan
}

(Received 20 September 2005 / Accepted 2 February 2006)

\begin{abstract}
In the Large Helical Device (LHD), of which the magnetic surfaces are surrounded by an ergodic magnetic field layer, the two-dimensional profile of electron density was for the first time measured by a newly developed sheet-shaped thermal lithium beam probe. The sheet beam was formed by guiding lithium vapor through three orifices placed in front of an aperture of an oven and injecting the vapor nearly vertically into the LHD plasma. The two-dimensional (2D) image of the LiI line was measured by using a CCD camera with an interference filter for LiI $(670.8 \mathrm{~nm})$. From the 2D image of LiI, we successfully obtained a two-dimensional profile of electron density in the plasma edge region.
\end{abstract}

(c) 2006 The Japan Society of Plasma Science and Nuclear Fusion Research

Keywords: two-dimensional electron density profile, lithium beam probe, a sheet beam, edge plasma

DOI: $10.1585 /$ pfr. 1.013

Detailed measurement and control of the edge plasma region are very important for tokamaks and helical devices to achieve a high confinement mode such as $\mathrm{H}$-mode where an edge transport barrier (ETB) is formed and is partially destroyed by so-called edge-localized modes (ELMs). Recently, ETB formation by LH transition was observed in LHD [1,2]. The magnetic configuration of LHD is threedimensional and has a helical divertor, where the nested magnetic surfaces are surrounded by a complicated ergodic magnetic field layer near the plasma edge [3]. The complicated edge structure is measured by using an accelerated lithium beam, and is partly clarified [4]. Twodimensional measurement of the edge plasma structure is necessary and effective for understanding edge plasma behavior in this magnetic configuration. For the purpose of two-dimensional measurement, we have developed a new type of lithium beam probing (LiBP) system using a sheet-shaped thermal beam and applied the system to lowdensity plasmas in LHD.

In this newly developed lithium beam probe, pure lithium ingot in an oven is heated to about $800 \mathrm{~K}$, and generated lithium vapor is conducted to an LHD plasma through three horizontally-elongated orifices. A sheetshaped thermal beam was successfully produced by this simple technique. The beam expansions in the direction of the major radius and toroidal direction are respectively about $680 \mathrm{~mm}$ and $50 \mathrm{~mm}$ on the mid plane of LHD, as shown in Fig. 1 (a). The energy of the beam is $\sim 0.07 \mathrm{eV}$, which corresponds to the beam velocity of

author'se-mail: y-taka@nifs.ac.jp
$1.4 \times 10^{3} \mathrm{~ms}^{-1}$. The expected spatial resolution is very good $\left(\sim 4.0 \times 10^{-5} \mathrm{~m}\right)$ because of the low beam velocity. A $2 \mathrm{D}$ image of the resonance line LiI $(670.8 \mathrm{~nm})$ emitted from the sheet-shaped beam is taken by a CCD camera along

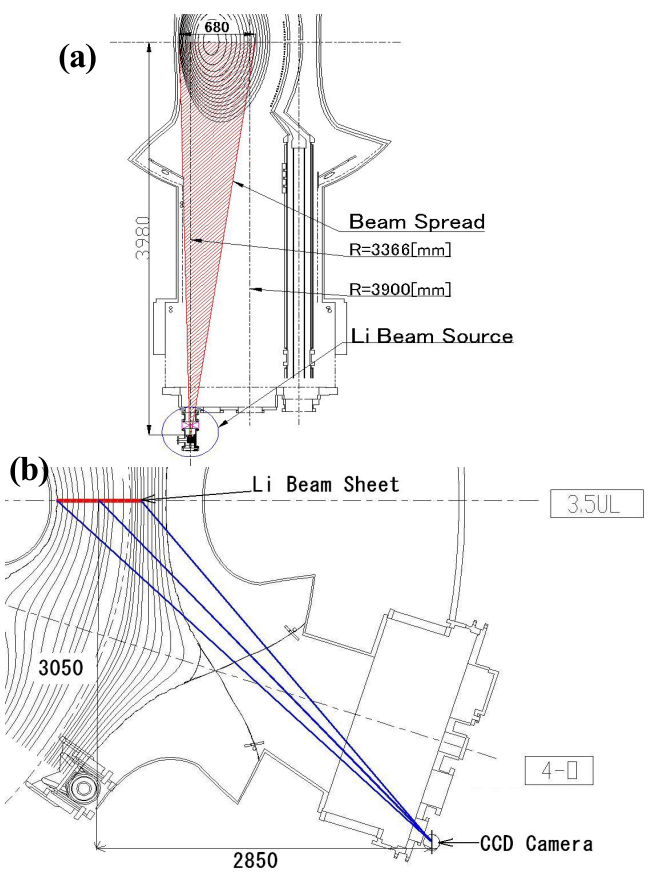

Fig. 1 (a) Vertical arrangement of the sheet-shaped Li beam on LHD, (b) Plan view of an LHD plasma where the sheetshaped beam and CCD camera are arranged. 


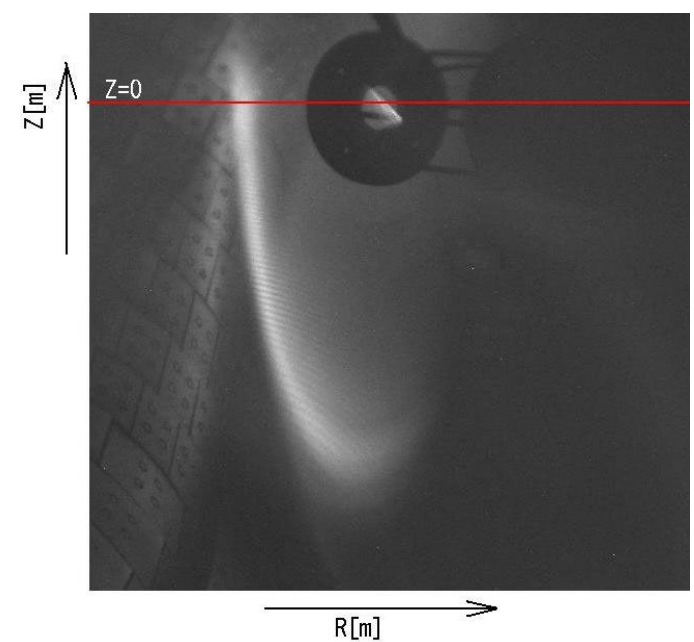

Fig. 2 Obtained 2D image of LiI emission, where the background image taken without $\mathrm{Li}$ beam injection was subtracted from the image taken in the case with the beam injection.

the viewing direction tilted to about 45 degrees against the lithium beam sheet, as shown in Fig. 1 (b).

In LHD, the sheet-shaped Li beam was applied to a fairly low density plasma of line averaged density $\left\langle n_{\mathrm{e}}\right\rangle \sim$ $0.5 \times 10^{18} \mathrm{~m}^{-3}$ produced by low-power electron cyclotron waves. A typical image of LiI taken by the CCD camera is shown in Fig. 2. It should be noted that the beam uniformity in this experiment was not good, that is, it was only $\sim \pm 20 \%$ along the beam sheet. If a beam source is placed far away from the LHD plasma, we will be able to assume that many pencil beams arranged along the major radius of the torus are injected vertically into the plasma. For simplicity, we introduce the above assumption, and derive a 2D profile of electron density from the LiI image shown in Fig. 2. Based on that assumption, an electron density profile along the Z-direction is derived for each pensile beam from the following simple equation:

$$
n_{\mathrm{e}}(Z)=\frac{v_{\mathrm{b}} I(Z)}{\langle\sigma v\rangle_{\mathrm{i}} \int_{Z}^{\infty} I(Z) \mathrm{d} Z}
$$

where $I(Z)$ is the observed profile of the LiI emission in the vertical direction, and $v_{\mathrm{b}}$ and $\langle\sigma v\rangle_{\mathrm{i}}$ are the beam velocity and ionization rate coefficient, respectively. Here, $\langle\sigma v\rangle_{\mathrm{i}}$ is assumed to be constant for the temperature range in the edge region, because the electron temperature in the edge region of LHD where this LiBP is applied is considered to

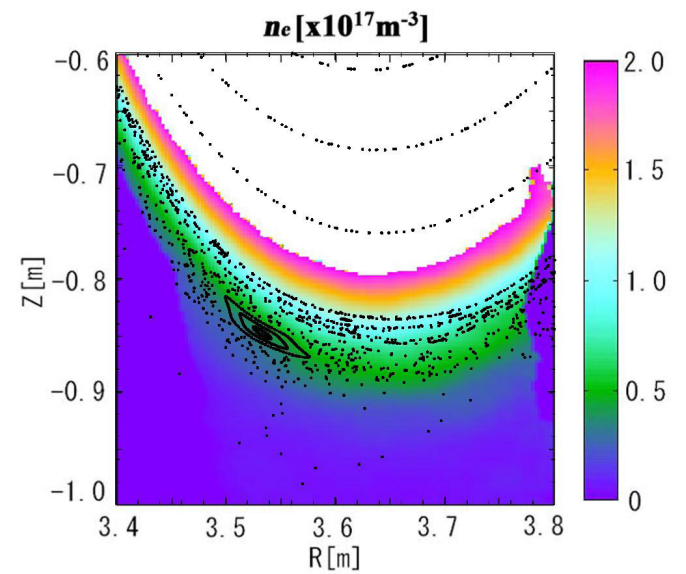

Fig. 3 Contour plot of 2D profile of electron density derived from LiI emission image of Fig. 2. Black dots indicate the magnetic surfaces and colored contours indicate electron density in $10^{17} \mathrm{~m}^{-3}$ unit.

be more than $10 \mathrm{eV}$ [5].

The 2D electron density profile from the data of Fig. 2 is shown in Fig. 3, where the profile is reconstructed up to $2.0 \times 10^{17} \mathrm{~m}^{-3}$. The profile is zoomed in the edge region around the lower null point of the divertor $(-1.0<$ $Z<-0.6 \mathrm{~m}, 3.4<R<3.8 \mathrm{~m}$ ). The electron density contours agree well with the magnetic surfaces in the region of $R<3.7 \mathrm{~m}$. In the more outer region of $R>3.75 \mathrm{~m}$, however, the density contours appreciably deviate from the magnetic surfaces. This may be caused by our analysis method based on the assumption of vertically injected pencil beams. Electron density rises from the outer boundary of the ergodic layer, having a finite density gradient.

In conclusion, this simple lithium beam probe technique based on a sheet-shaped thermal beam has been successfully applied to an LHD plasma edge region and demonstrated high potentiality for two-dimensional study of the edge plasma region including the divertor region.

This work is supported in part by the Grant-in-Aid for Scientific Research (A) from JSPS, No.15206107, and NIFS05ULPP507.

[1] K. Toi et al., Nucl. Fusion 44, 217 (2004).

[2] K. Toi et al., Phys. Plasmas 12, 020701-1 (2005).

[3] T, Morisaki et al., J. Nucl. Mater. 313-316, 548 (2003).

[4] T. Morisaki et al., Rev. Sci. Instrum. 73, 1865 (2003).

[5] K. Kadota et al, J. Nucl. Mater. 128-129, 960 (1984). 